NOTE

\title{
A modification of the Bohr method to determine airways deadspace for non-uniform inspired gas tensions
}

\author{
Phi Anh Phan', C E W Hahn ${ }^{1}$, and A D Farmery ${ }^{1}$
}

1 Nuffield Division of Anaesthetics, University of Oxford, John Radcliffe Hospital, Oxford, OX3 9DU

Email: Phi Anh Phan (+44-18-6523-1420, phi.phan@nda.ox.ac.uk);

Clive E.W. Hahn( clive.hahn@nda.ox.ac.uk)

Andrew Farmery ( 


\begin{abstract}
Background The Bohr method is a technique to determine airways deadspace using a tracer gas such as carbon dioxide or nitrogen. It is based on the assumption that the inspired concentration of the tracer gas is constant throughout inspiration. However, in some lung function measurement techniques where inspired concentration of the tracer gas may be required to vary, or where rapid injection of the tracer gas is made in real time, uniform inspired concentration is difficult or impossible to achieve, which leads to inaccurate estimation of deadspace using the Bohr equation. One such lung function measurement technique is the inspired sinewave technique. Objective In this paper, we proposed a modification of the Bohr method, relaxing the requirement of absolute uniformity of tracer concentration in the inspired breath. Method. The new method used integration of flow and concentration. A computer algorithm sought an appropriate value of deadspace to satisfy the mass balance equation for each breath. A modern gas mixing apparatus with rapid mass flow controllers was used to verify the procedure. Result Experiments on a tidally ventilated bench lung showed that the new method estimated dead space within $10 \%$ of the actual values whereas the traditional Bohr deadspace gave more than 50\% error. Conclusion The new method improved the accuracy of deadspace estimation when the inspired concentration is not uniform. This improvement would lead to more accurate diagnosis and more accurate estimations of other lung parameters such as functional residual capacity and pulmonary blood flow.
\end{abstract}

\title{
1 Introduction
}

Respiratory dead space is an important parameter regarding the efficiency of ventilation and its relation to pulmonary perfusion [1], [2], with relevance in respiratory physiology, clinical anesthesia, and critical care medicine. A non-zero difference between physiologic and anatomical dead spaces is a result of both acinar and conductive ventilation heterogeneity [3]. A high ratio of physiologic dead space to tidal volume is associated with increase mortality rate in infants with congenital diaphragmatic hernia [4], critical ill children with lung injury [5], and adults with acute respiratory distress syndrome [6]. It is therefore important to accurately estimate respiratory dead space.

The Bohr method is the traditional method to determine anatomical airways deadspace. It is based on the assumption that the concentration of the indicator gas in the inspired air is constant throughout inspiration. $\mathrm{CO}_{2}$ is the most common indicator gas used to determine deadspace, but in some cases, other indicator gases such as nitrogen or oxygen have been used [7], [8]. In other techniques, the concentration of indicator in the inspired gas can be made to vary between breaths such as the sinusoidally varying inspired oxygen tensions employed by Williams and colleagues [8]. These inspired gas variations may be achieved by pre-mixing the inspired gas breath by breath, or, in other cases by using devices that allow rapid injection of indicator gases [9]-[12] into the inspired airflow in real time. In these circumstances, uniform inspired concentrations are difficult or impossible to achieve, which leads to inaccurate estimations of deadspace. This may be due to hardware limitations including physical limitations of the mass flow controllers, and the time delay in the feedback control loop, as well as inadequate gas mixing.

This problem is even more marked when these devices are used in mechanically ventilated subjects in whom the flow pattern changes more abruptly than in spontaneous breathing. Figure 1 demonstrates the non-uniformity of the inspired concentration of the indicator gas in an inspired sinewave gas mixing device. Even though it might be possible to overcome this limitation by improving the hardware performance, a more pragmatic and flexible approach is to solve it analytically by using a more robust algorithm to estimate dead space. 


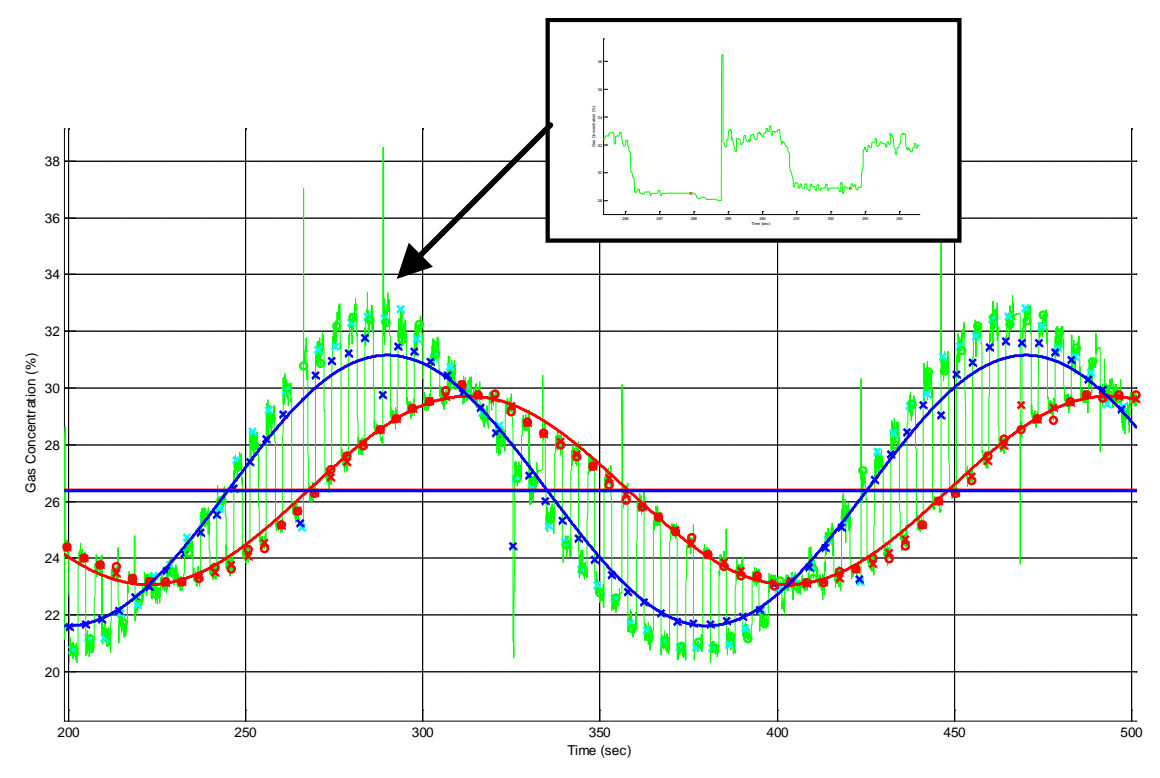

Figure 1. An example of $\mathrm{O}_{2}$ concentration signal collected in an inspired sinewave lung function test. Green line is the measured $\mathrm{O}_{2}$ signal. Blue marker and line are the mixed inspired concentration and sinewave. Red marker and line are the end-expired concentration and sinewave. The sub-figure is the zoomed-up concentration signal showing the non-uniformed inspired concentration.

Consequently, the objective of this paper is to propose a modification of the Bohr method using reverse integration to relax the requirement of absolute uniformity of tracer concentration in the inspired breaths. The method uses integration of flow and concentration to compute the molar masses of inspired gases in any given point in time. A computer algorithm then seeks an appropriate value of deadspace to satisfy the mass balance equation for each breath. This method is primarily aimed at devices in which constant inspired concentrations are not guaranteed. For constant inspired concentrations, the proposed method would give the same result as the traditional Bohr method. In this sense, this new method can be considered as a generalisation of the Bohr deadspace equation. This new method is also described in a recent patent application [13].

The rest of the paper is organised as follows. Section 2.1 re-visits the Bohr method then presents the reverse integration method. Section 2.2 describes the hardware used to test the method. Section 3 details the experimental results on a mechanical bench lung. Section 4 gives discussion and conclusion, including recommended performance of flow and concentration sensors to achieve a desirable accuracy.

\section{Method and Hardware}

\subsection{Method}




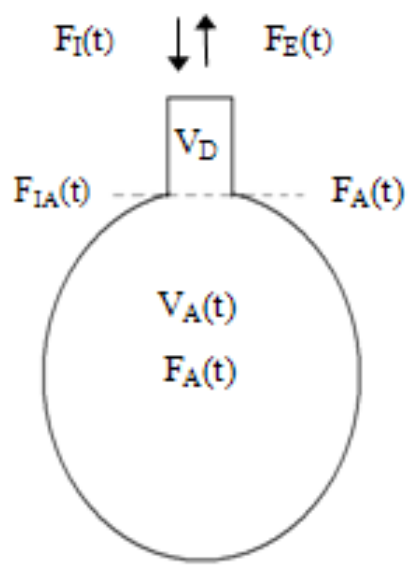

Figure 2. A tidally ventilated "balloon on a straw" model is considered for the analysis of dead space $V_{D}$. $F_{I}(t)$ is the inspired concentration as a function of time t. $F_{I A}(t)$ is the inspired concentration "seen" by the alveolar at time t. $\mathrm{V}_{\mathrm{A}}(\mathrm{t})$ and $\mathrm{F}_{\mathrm{A}}(\mathrm{t})$ are the volume and concentration of the alveolar respectively. $\mathrm{F}_{\mathrm{E}}(\mathrm{t})$ is the expired concentration. The dead space is defined as the portion of inspired gas that passes the lips but never gets to the alveolar compartment.

The lung is considered as a tidally ventilated "balloon on a straw" [14], [15] shown in Figure 2. During inspiration, the inspired gas with concentration $F_{I}(t)$ travels through the dead space of volume $V_{D}$ to get to the alveolar compartment. Due to the transport delay through the dead space, the inspired gas concentration "seen" by the lung $\mathrm{F}_{\mathrm{IA}}(\mathrm{t})$ is different from $\mathrm{F}_{\mathrm{I}}(\mathrm{t})$ measured at the lips. During expiration, the alveolar gas of concentration $\mathrm{F}_{\mathrm{A}(\mathrm{t})}$ travels through the dead space past the lips where its final value is $\mathrm{F}_{\mathrm{E}}(\mathrm{t})$. The dead space is defined as the portion or fraction of inspired gas that passes the lips but does not access the alveolar compartment. First, we will re-visit the Bohr method. Then, we will extend to the proposed robust reversed-integration method to estimate dead space.

\subsubsection{Bohr method}

The Bohr method assumes that the inspired concentration of the indicator gas is uniform so that $F_{I}(t)=$ $F_{I A}(t)=F_{I}$. From Figure 2, at the end of an inspiration, the concentration in the dead space is $F_{I}$. During expiration, the mass of tracer exhaled equals that lost from the dead space in the initial portion of the expirate, and that lost from the alveolar compartment in the later portion of the expirate. This is expressed as:

$$
V_{T} \times F_{\bar{E}}=F_{I} \times V_{D}+F_{A} \times\left(V_{T}-V_{D}\right)
$$

where $V_{T}$ is the tidal volume and $F_{\bar{E}}$ is the mixed-expired concentration.

Manipulating (1) gives the general Bohr equation:

$$
V_{D}=V_{T} \frac{F_{A}-F_{\bar{E}}}{F_{A}-F_{I}}
$$

For the special case of $\mathrm{CO}_{2}$, since there is no or little $\mathrm{CO}_{2}$ in inspired gas, there is no or little $\mathrm{CO}_{2}$ in the dead space at the end of an inspiration, i.e. $F_{I, C O 2}=0$. The Bohr dead space equation therefore becomes [1], [15]:

$$
V_{D, \mathrm{CO} 2}=V_{T} \frac{F_{A, \mathrm{CO} 2}-F_{\bar{E}, \mathrm{CO} 2}}{F_{A, \mathrm{CO} 2}}
$$

\subsubsection{A robust method: reversed-integration}



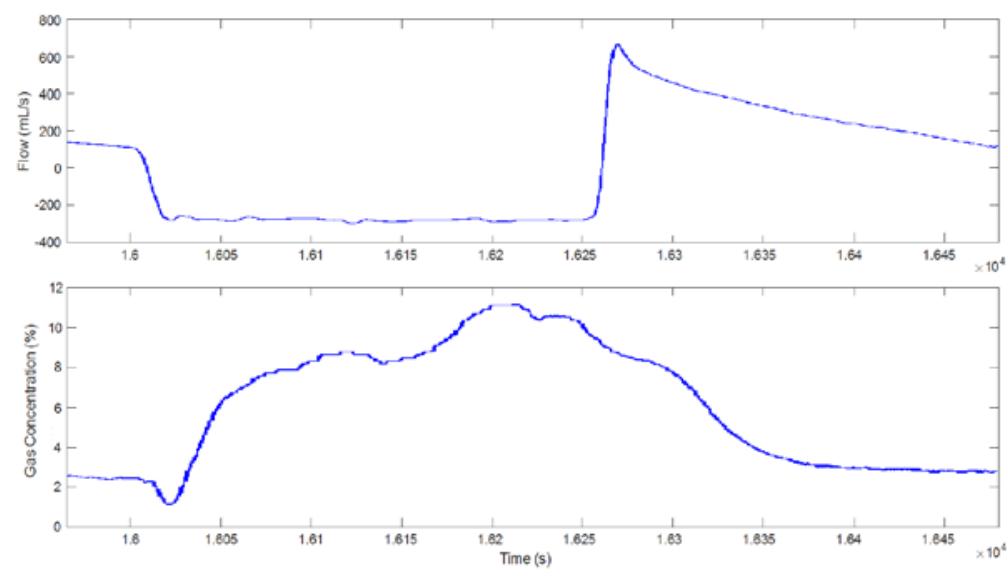

(a) Flow and concentration

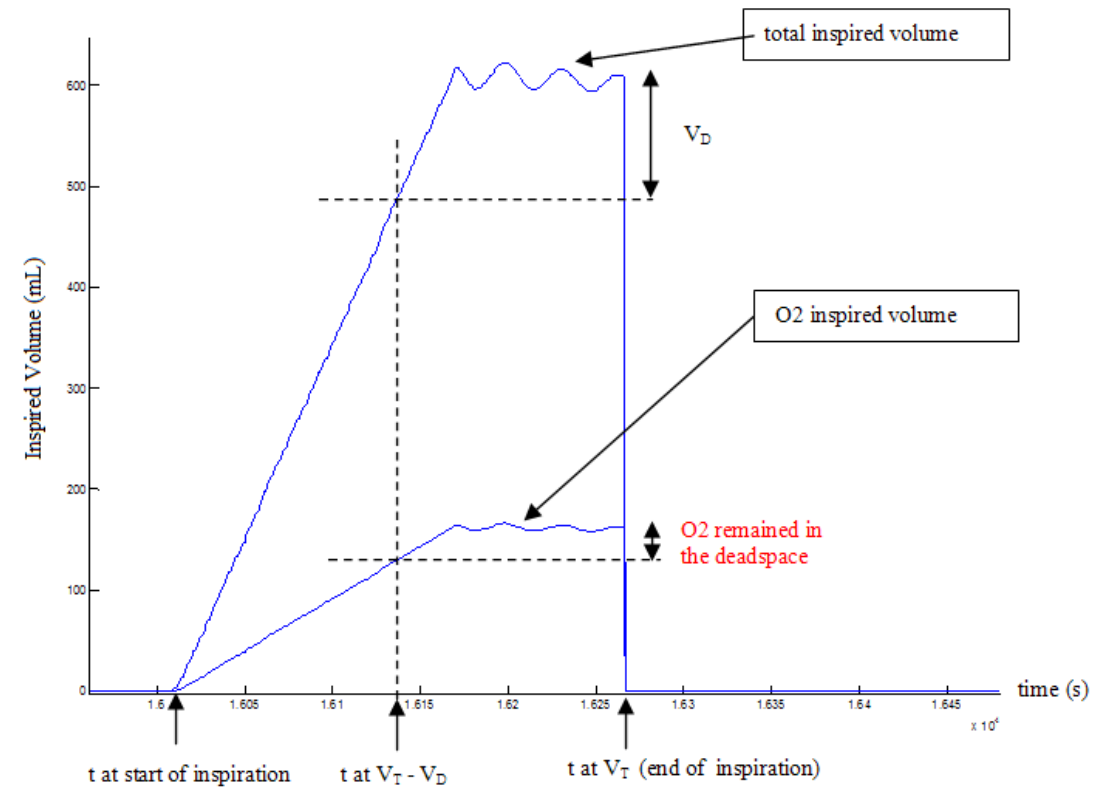

(b) The total and $\mathrm{O} 2$ inspired volumes during one inspiration

Figure 3 An example of one inspiration. The total and $\mathrm{O} 2$ inspired volumes increase from the start of the inspiration up to time equals to $V_{T}-V_{D}$. After that, the inspired volumes from the time at $V_{T}-V_{D}$ to the end of inspiration serves only to fill the deadspace $V_{D}$ and none of this gas portion gets to the alveolus. The corresponding $\mathrm{O}_{2}$ inspired volume during that same period, from $t=V_{T}-V_{D}$ to the end of inspiration, is the $\mathrm{O}_{2}$ remained in the dead space.

When the concentration of the inspired indicator gas is non-uniform, such as shown in Figure 1, the total inspired volume and inspired indicator volume that pass the lips during an inspiration are calculated respectively as:

$$
\text { total inspired gas volume }=\int_{t_{\text {insp }}}^{t} \dot{V}_{T}(t) \times d t
$$

and

$$
\text { inspired indicator gas volume }=\int_{t_{\text {insp }}}^{t} F_{I}(t) \times \dot{V}_{T}(t) \times d t
$$

where $t_{\text {insp }}$ is the time at the start of inspiration, $\dot{V}_{T}(t)$ is the inspired flow rate. An example of these 2 functions is demonstrated in Figure 3. 
The indicator gas remaining in the deadspace at the end of $i$-th inspiration is:

$$
\text { indicator gas remained in the dead space } \mathrm{i}_{\mathrm{i}-\mathrm{th}}=\int_{t_{V T-V D}}^{t_{V T}} F_{I}(t) \times \dot{V}_{T}(t) \times d t
$$

where $t_{V T-V D}$ is the time after which any gas that passes the lips only fills the dead space, $t_{V T}$ is the time at the end of the inspiration. With $V_{D}$ unknown, (6) can be interpreted as a function of dead space where $V_{D}$ can take a value between 0 and $V_{T}$ :

$$
f\left(V_{D}\right)=\int_{t_{V T-V D}}^{t_{V T}} F_{I}(t) \times \dot{V}_{T}(t) \times d t
$$

As the indicator gas remaining in the dead space at the end of an inspiration will go out in the next expiration, the mass balance eq (1) becomes:

$$
\begin{aligned}
& F_{\bar{E}} \times V_{T}=f\left(V_{D}\right)+F_{A} \times\left(V_{T}-V_{D}\right), \text { which rearranges to } \\
& f\left(V_{D}\right)-F_{A} \times V_{D}=\left(F_{\bar{E}}-F_{A}\right) \times V_{T}
\end{aligned}
$$




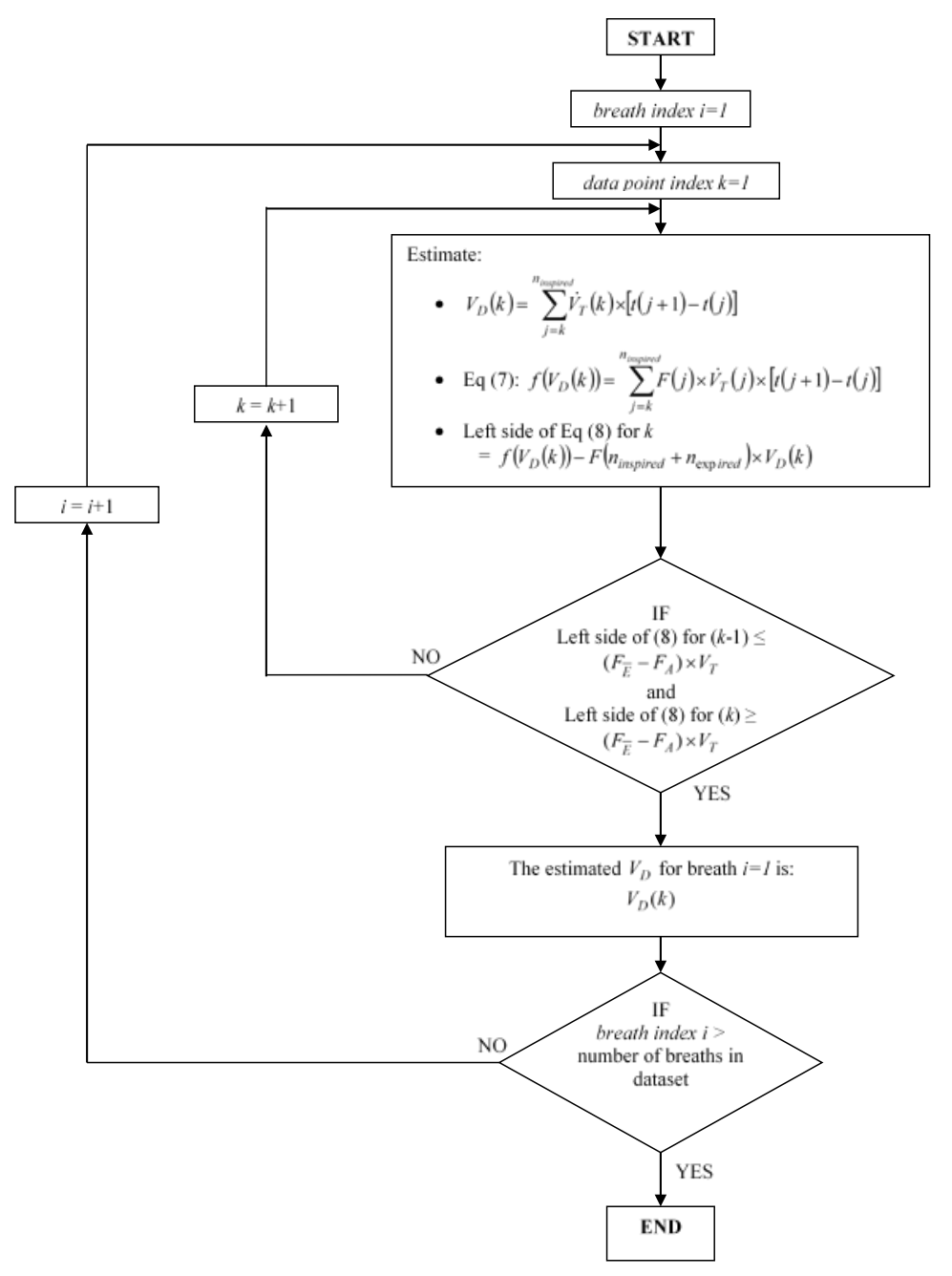

Figure 4 The algorithm used to find deadspace $V_{D}$ for each breath from eq (6), (7), (8).

Eq (6), (7), and (8) then can be solved to obtain $V_{D}$ using the algorithm described in Figure 4. For each breath, the algorithm first estimates numerical values of the left sides of eq (6), (7), (8) for $V_{D}$ values between $\left[0, V_{T}\right]$, i.e all available data points. It then finds the value of $V_{D}$ that gives the least error in (8). This value is the computational solution of deadspace $V_{D}$.

The proposed method is valid only if the sensors are sufficiently fast and accurate to allow integrations (4) and (5). To verify this, we set up an experiment with a real time inspired gas mixing apparatus, and a mechanical bench lung. The mixing apparatus is designed for the inspired sinewave technique, but it can be used to deliver any desirable pattern of inspired concentration. The results are given in the next section.

2.2 Hardware: the inspired gases mixing apparatus and the experiment setup 


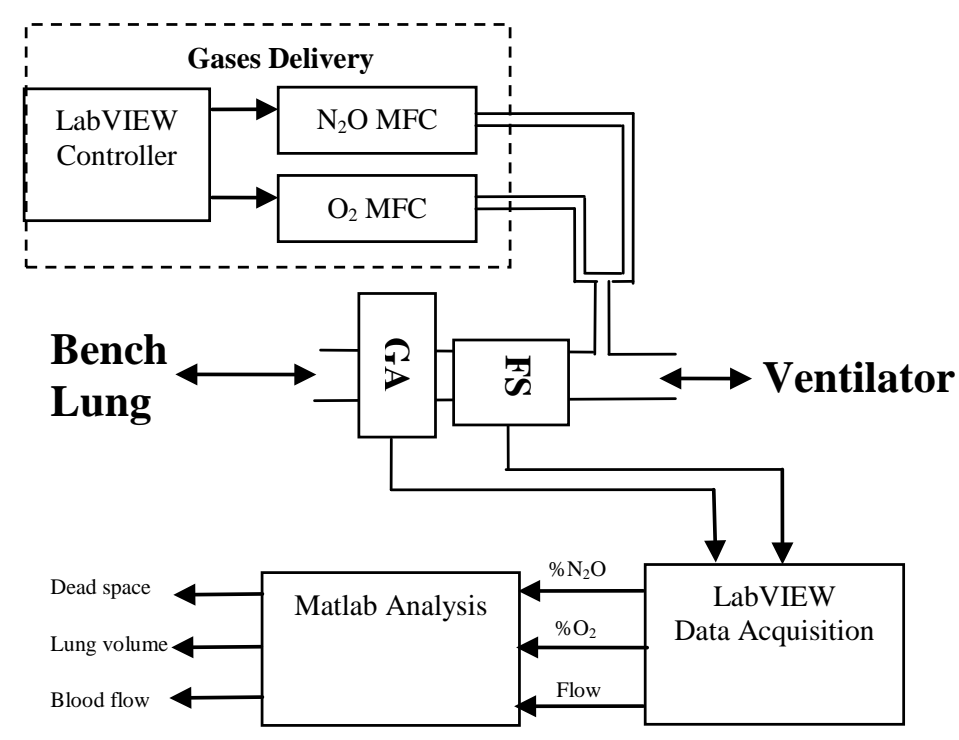

Figure 5 Layout of the Inspired Sinewave Device. The gases delivery system employs mass flow controllers (MFCs) to inject $\mathrm{O}_{2}$ and $\mathrm{N}_{2} \mathrm{O}$ into the breathing circuit. Real-time data is read by the flow sensor (FS) and the mainstream gas analyser (GA) and fed to LabVIEW and Matlab for estimation of dead space, lung volume and pulmonary blood flow.

\subsubsection{The inspired gases mixing apparatus}

The inspired concentration was generated by a gas mixing apparatus depicted in Figure 5. By reading the inspiratory flow rate in real time through a flow sensor (FS), the LabVIEW software estimated the desired amount of indicator gas (oxygen in this case) needed to achieve the desired inspired concentration. The software then sent the desired set-point to the mass flow controller (MFC) to inject indicator gas "on the fly" into the inspired breath. A gas analyser (GA) was used to measure the actual concentration of the indicator gas in the inspired breath.

The gas analyser (Phasein Technology, Masimo Sweden AB) simultaneously measured main-stream CO2, $\mathrm{N} 2 \mathrm{O}$, and $\mathrm{O} 2$ concentrations. The analyser utiltised non - dispersive infrared technology to measure $\mathrm{CO} 2$ and $\mathrm{N} 2 \mathrm{O}$ concentrations. To measure $\mathrm{O} 2$ concentration, it utilized the fuel cell technology. The gas analyser had an accuracy of $2 \%$ of the reading and a step response time of less than $100 \mathrm{~ms}$, and $5 \mathrm{~ms}$ time delay. With software compensation, the time alignment between the gas analyser and flow sensor was kept under 10ms, as recommended by the American Thoracic Society and the European Respiratory Society [16].

The flow sensor (VenThor-22/2A, Hungary) was an ultrasonic flow meter, with an updating rate of $50 \mathrm{~Hz}$ and accuracy of $+/-2.5 \%$ reading.

\subsubsection{The experimental setup}

To test the Bohr and the proposed methods, a mechanical water-displacement bench lung (Figure 6) was used [17]. The inspired gases mixing apparatus was connected to the bench lung, which was tidally ventilated by an Elema-Schonander 900 ventilator. The advantage of using this bench lung instead of a human subject was that we could set the exact geometry for lung dead space and alveolar volume. The alveolar volume was determined by setting the water level in the bench lung. The deadspace was set by adding extended tubes to the original deadspace tubes showed in Figure 5(b). Therefore, we could evaluate the Bohr and the proposed methods against exactly known volumes. 
For our purpose, we performed two experiments. The first experiment used three configurations of dead space (108ml; 208ml; and $258 \mathrm{ml}$ ) and an alveolar volume of $2.6 \mathrm{~L}$ to evaluate the deadspace estimation. The second experiment used a deadspace of $208 \mathrm{ml}$ and three configurations of alveolar volume (1.8L; 2.2L; and 2.6L) to evaluate the alveolar volume estimation. For each configuration, we collected 60 breathing data sets, giving 300 breathing data sets for analysis. 




(a)



(b)

Figure 6 The mechanical water-displacement bench lung used to test deadspace methods. (a) The 'alveolar volume' was set by filling up the water in the large cylinder. Tidal breathing was created by letting the tidal gas flow from a ventilator into the larger cylinder during inspiration. (b) The deadspace was constructed using multiple parallel tubes to minimise the diffusion effect.

\section{Results}

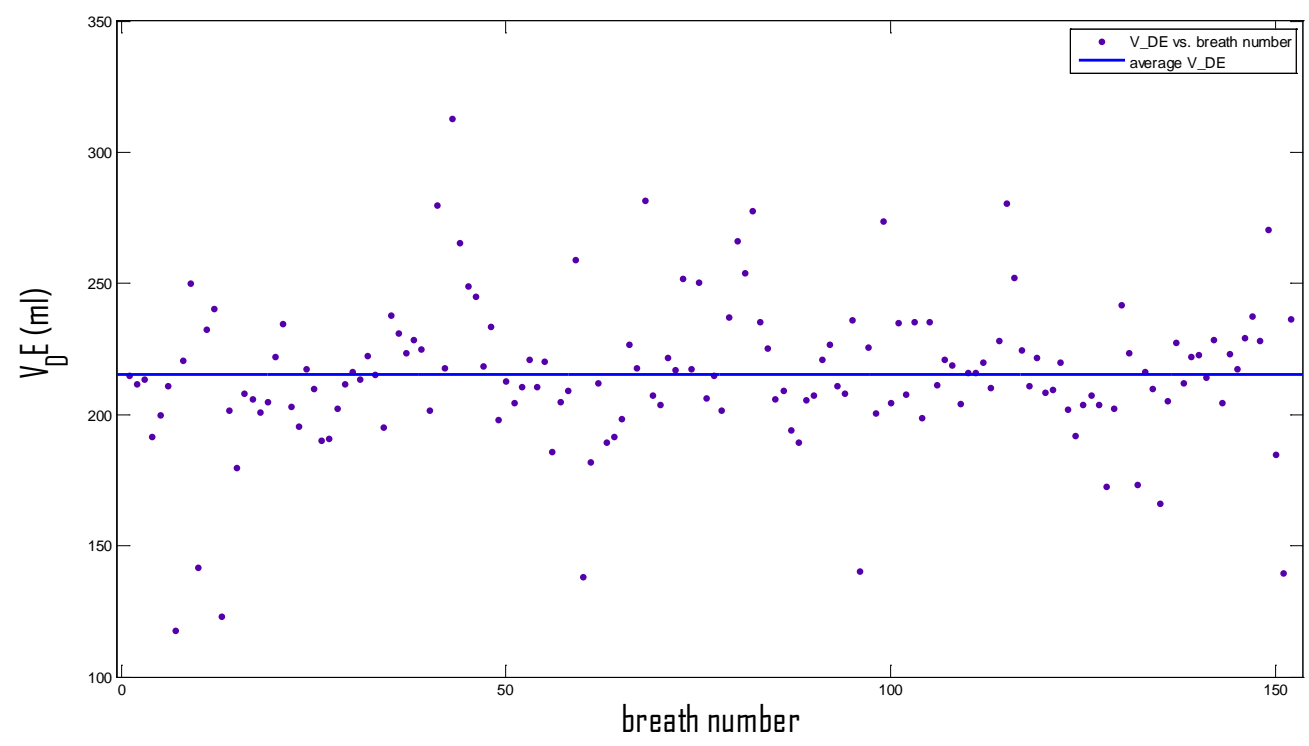

Figure 7. An example of dead space estimation for one breathing dataset. Markers are values of dead space estimated in individual breaths. A Matlab fitting algorithm removes outliers and computes the average dead space, displayed as the blue line. 
Figure 7 demonstrates the dead space estimation of one breathing data set. For each breath, an estimation of dead space using eq (7) and (8) was computed and represented as a marker on the figure. For the entire breathing dataset of more than 100 breaths, the Matlab fitting function fit() with 'LinearLeastSquare' and 'Robust' options was used to removed outliers and computed the average dead space.

In the first experiment deadspace was varied from $108 \mathrm{ml}$, to $208 \mathrm{ml}$, and then to $258 \mathrm{ml}$ while alveolar volume was fixed at $2.6 \mathrm{~L}$. For each data set, the data was collected for duration of 12 mins. The ventilator settings were $0.6 \mathrm{~L}$ tidal volume and $12 \mathrm{breaths} / \mathrm{min}$. The results were given in Table 1 . It could be seen that the proposed method out-performed the Bohr method, with errors of less than $10 \%$ for dead space and $6 \%$ for alveolar volume.

In the second experiment, alveolar volume was varied from 1.8L, 2.2L, to 2.6L while deadspace was fixed at $208 \mathrm{ml}$. The results are given in Table 2 . The 95\% confidence ranges are less than $15 \mathrm{ml}$ and $0.2 \mathrm{~L}$ for dead space and alveolar volume respectively. It should be noted that an algorithm to estimate alveolar volume is not described in this paper, but a few methods have been described in [18]-[20]. Evaluating estimations of alveolar volume was included to emphasise that incorrect estimations of dead space would lead to incorrect estimations of other lung parameters, and vice versa.

\section{EXPERIMENT 1 -DEAD SPACE ESTIMATION}

\begin{tabular}{|l|c|c|c|}
\hline & Setup 1 & Setup 2 & Setup 3 \\
\hline Deadspace & $108 \mathrm{ml}$ & $208 \mathrm{ml}$ & $258 \mathrm{ml}$ \\
\hline Alveolar Volume & $2.6 \mathrm{~L}$ & $2.6 \mathrm{~L}$ & $2.6 \mathrm{~L}$ \\
\hline
\end{tabular}

(a) Actual bench lung parameters

\begin{tabular}{|l|c|c|c|}
\hline & Setup 1 & Setup 2 & Setup 3 \\
\hline Deadspace (SD) & $228(13) \mathrm{ml}$ & $327(5) \mathrm{ml}$ & $393(6.5) \mathrm{ml}$ \\
\hline \% Deadspace Error & $111(12) \%$ & $57.2(2.0) \%$ & $52.3(3.0) \%$ \\
\hline Alveolar Volume (SD) & $1.83(0.135) \mathrm{L}$ & $1.52(0.065) \mathrm{L}$ & $1.31(0.075) \mathrm{L}$ \\
\hline \% Alveolar Volume Error & $-77(5.0) \%$ & $-41.5(2.5) \%$ & $-49.6(2.9) \%$ \\
\hline
\end{tabular}

(b) Estimations using Bohr method

\begin{tabular}{|l|c|c|c|}
\hline & Setup 1 & Setup 2 & Setup 3 \\
\hline Deadspace & $110(5) \mathrm{ml}$ & $206(4) \mathrm{ml}$ & $260(4) \mathrm{ml}$ \\
\hline \% Deadspace Error & $1.8(4.6) \%$ & $-1.0(1.9) \%$ & $0.8(1.6) \%$ \\
\hline Alveolar Volume & $2.71(0.07) \mathrm{L}$ & $2.72(0.07) \mathrm{L}$ & $2.48(0.08) \mathrm{L}$ \\
\hline \% Alveolar Volume Error & $4.2(2.7) \%$ & $4.6(2.3) \%$ & $-4.6(3.1) \%$ \\
\hline
\end{tabular}

(c) Estimations using the proposed method

Table 1 Experiment 1 results. Values are mean(SD). It can be seen that the proposed method was more accurate and robust, with estimations of deadspace within $15 \mathrm{ml}$ of expected values, whereas the Bohr method gave error larger than $100 \mathrm{ml}$.

\section{Conclusion}

This paper describes a new method to estimate airways dead space to be used with any lung function technique or device where inspired gases are mixed in real time, i.e. directly injected into the inspired breaths. It uses an integration method to relax the assumption that the inspired gas concentration must be uniform during an entire inspiration. Our experiments show that the proposed method estimates dead space within $10 \%$ of expected values, whereas the traditional Bohr method gives more than $50 \%$ error. 
EXPERIMENT 2-LUNG VOLUME ESTIMATION

\begin{tabular}{|l|c|c|c|}
\hline & Setup 4 & Setup 5 & Setup 6 \\
\hline Deadspace & $208 \mathrm{ml}$ & $208 \mathrm{ml}$ & $208 \mathrm{ml}$ \\
\hline Alveolar Volume & $1.8 \mathrm{~L}$ & $2.2 \mathrm{~L}$ & $2.6 \mathrm{~L}$ \\
\hline
\end{tabular}

(a) Actual bench lung parameters

\begin{tabular}{|l|c|c|c|}
\hline & Setup 4 & Setup 5 & Setup 6 \\
\hline Deadspace (SD) & $328(7) \mathrm{ml}$ & $331(7) \mathrm{ml}$ & $327(5) \mathrm{ml}$ \\
\hline \% Deadspace Error & $57.7(3.3) \%$ & $59.1(3.3) \%$ & $57.2(2.4) \%$ \\
\hline Alveolar Volume (SD) & $0.83(0.08) \mathrm{L}$ & $1.18(0.08) \mathrm{L}$ & $1.52(0.065) \mathrm{L}$ \\
\hline \% Alveolar Volume Error & $-53.9(4.4) \%$ & $-46.4(3.6) \%$ & $-41.5(2.5) \%$ \\
\hline
\end{tabular}

(b) Estimations using Bohr method

\begin{tabular}{|l|c|c|c|}
\hline & Setup 4 & Setup 5 & Setup 6 \\
\hline Deadspace(SD) & $210(7) \mathrm{ml}$ & $202(5) \mathrm{ml}$ & $206(4) \mathrm{ml}$ \\
\hline \% Deadspace Error & $1.0(3.4) \%$ & $-2.9(2.4) \%$ & $-1.0(1.9) \%$ \\
\hline Alveolar Volume (SD) & $1.86(0.09) \mathrm{L}$ & $2.17(0.075) 5 \mathrm{~L}$ & $2.72(0.07) \mathrm{L}$ \\
\hline \% Alveolar Volume Error & $3.3(5) \%$ & $-1.4(3.4) \%$ & $4.6(2.3) \%$ \\
\hline
\end{tabular}

(c) Estimations using the proposed method

Table 2 Experiment 2 results. Values are mean (SD).

In a broader sense, the experiments in this paper verify that the commercial gas and flow sensors are sufficiently fast to allow such integrations. This was not always possible in the past due to the slow response and variable time delay of older-generation sensors and actuators. The proposed algorithm therefore can be used by other gas mixing techniques such as [9], [10], [21].

It is recommended that the proposed method to be used with gas and flow sensors with similar performances to ours: $2 \%$ accuracy of reading, $100 \mathrm{~ms}$ step response time, $5 \mathrm{~ms}$ time delay for the gas sensor, and an update rate of $50 \mathrm{~Hz}$ and accuracy of $+/-2.5 \%$ reading for the flow sensor. Time alignment between the gas and flow sensors should be equal or less than 10ms, as recommended by the American Thoracic Society and the European Respiratory Society [16].

When implementing the method, care should be taken regarding different operating modes and conditions. If pressure-controlled ventilation is used, decelerating inspiratory flow would likely increase the nonuniformity of the inspired concentration. If lung-diseased subjects are tested, the abnormal airway would likely to affect both the proposed method and the traditional Bohr method. Moreover, a different hardware system, with different accuracy, time response, alignment, would likely result in a different estimation. A thorough validation test is therefore recommended.

\section{References}

[1] Y. Tang, M. J. Turner, and A. B. Baker, 'A New Equal Area Method to Calculate and Represent Physiologic, Anatomical, and Alveolar Dead Spaces’, Anesthesiology, vol. 104, no. 4, 2006.

[2] Y. Tang, M. J. Turner, and A. B. Baker, 'Effects of Alveolar Dead-Space, Shunt and V/Q Distribution on Respiratory Dead-Space Measurements', Br. J. Anaesth., vol. 95, no. 4, pp. 538548, Jan. 2005. 
[3] S. Verbanck, M. Paiva, D. Schuermans, S. Hanon, W. Vincken, and A. V. Muylem, 'Relationships between the lung clearance index and conductive and acinar ventilation heterogeneity’, J Appl Physiol, vol. 112, no. 5, pp. 782-790, Jan. 2012.

[4] J. H. Arnold, L. K. Bower, and J. E. Thompson, 'Respiratory deadspace measurements in neonates with congenital diaphragmatic hernia’, Crit. Care Med., vol. 23, no. 2, pp. 371-375, Feb. 1995.

[5] J. A. Coss-Bu, D. L. Walding, Y. B. David, and L. S. Jefferson, 'Dead space ventilation in critically ill children with lung injury’, Chest, vol. 123, no. 6, pp. 2050-2056, Jun. 2003.

[6] T. J. Nuckton et al., 'Pulmonary dead-space fraction as a risk factor for death in the acute respiratory distress syndrome’, N. Engl. J. Med., vol. 346, no. 17, pp. 1281-1286, Apr. 2002.

[7] W. S. Fowler, 'Lung function studies; the respiratory dead space.', The American journal of physiology, vol. 154, no. 3, pp. 405-16, 1948.

[8] E. M. Williams, R. M. Hamilton, L. Sutton, J. P. Viale, and C. E. W. Hahn, 'Alveolar and Dead Space Volume Measured by Oscillations of Inspired Oxygen in Awake Adults’, Am. J. Respir. Crit. Care Med., vol. 156, no. 6, pp. 1834-1839, Dec. 1997.

[9] I. Gültuna, P. E. M. Huygen, C. Jabaaij, W. P. J. Holland, C. Ince, and H. A. Bruining, 'A simple device to inject indicator gas for wash-out tests during mechanical ventilation', Intensive Care Medicine, vol. 18, no. 5, pp. 304-308, 1992.

[10] M. Kirmse, D. Hess, Y. Fujino, R. M. Kacmarek, and W. E. Hurford, 'Delivery of Inhaled Nitric Oxide Using the Ohmeda INOvent Delivery System’, Chest, vol. 113, no. 6, pp. 1650-1657, Jun. 1998.

[11] J. K. K. Kanhai, P. J. Caspers, E. G. J. Reinders, J. C. Pompe, H. A. Bruining, and G. J. Puppels, 'A fast, digitally controlled flow proportional gas injection system for studies in lung function', Biomedical Engineering, IEEE Transactions on, vol. 50, no. 11, pp. 1268-1275, Nov. 2003.

[12] L. Clifton, A. D. Farmery, and C. C. W. Hahn, 'Assessment of Lung Function using a Noninvasive Oscillating Gas-forcing Technique’, in publication, 2012.

[13] P. A. Phan, A. D. Farmery, and C. E. W. Hahn, 'Method and Apparatus for Measurement of Cardiopulmonary Function’, P41221GB, Mar-2014.

[14] C. E. W. Hahn and A. D. Farmery, 'Gas exchange modelling: no more gills, please', British Journal of Anaesthesia, vol. 91, no. 1, pp. 2-15, Jul. 2003.

[15] J. B. West, Respiratory Physiology: The Essentials. Lippincott Williams \& Wilkins, 2008.

[16] P. D. Robinson et al., 'Consensus statement for inert gas washout measurement using multipleand single- breath tests', Eur. Respir. J., vol. 41, no. 3, pp. 507-522, Mar. 2013.

[17] E. M. Williams, L. B. Gale, P. A. Oakley, M. C. Sainsbury, and C. E. Hahn, 'Development of a concentric water-displacement model lung’, J Biomed Eng, vol. 15, no. 5, pp. 420-424, Sep. 1993. 
[18] D. J. Gavaghan and C. E. W. Hahn, 'A tidal breathing model of the forced inspired inert gas sinewave technique’, Respiration Physiology, vol. 106, no. 2, pp. 209-221, Nov. 1996.

[19] J. P. Whiteley, D. J. Gavaghan, and C. E. W. Hahn, 'A tidal breathing model of the inert gas sinewave technique for inhomogeneous lungs', Respiration Physiology, vol. 124, no. 1, pp. 6583, Dec. 2000.

[20] L. Clifton, A. D. Farmery, and C. E. W. Hahn, 'A novel non-invasive method for estimating cardiopulmonary variables using breath-by-breath tracer gas injection’, in publication, 2012.

[21] J. K. K. Kanhai, P. J. Caspers, E. G. J. Reinders, J. C. Pompe, H. A. Bruining, and G. J. Puppels, 'A fast, digitally controlled flow proportional gas injection system for studies in lung function', Biomedical Engineering, IEEE Transactions on, vol. 50, no. 11, pp. 1268-1275, Nov. 2003. 